\title{
Thermal-flow technique for reducing surface roughness and controlling gap size in polymer microring resonators
}

\author{
Chung-Yen Chao and L. Jay Guo a) \\ Solid-State Electronics Laboratory, Department of Electrical Engineering and Computer Science, \\ University of Michigan, Ann Arbor, Michigan 48109
}

(Received 9 June 2003; accepted 4 February 2004)

\begin{abstract}
$Q$ factors of microring resonator waveguide devices are primarily limited by the surface-roughness-induced scattering loss. Such surface roughness loss has been observed in waveguides that are fabricated from various types of materials, including semiconductors, dielectrics, and polymers. In this letter, we show that the surface roughness of polymer waveguides can be greatly reduced by a thermal-flow technique, and the effective reduction in waveguide loss was verified experimentally. In addition to smoothing surfaces, this technique can simultaneously shrink the gap distance in the coupling region of polymer microring resonators. This, in turn, provides higher coupling, lessens the difficulty of fabricating submicron gaps, and leads to more compact devices. (C) 2004 American Institute of Physics. [DOI: 10.1063/1.1691492]
\end{abstract}

Microring-resonator-based photonic devices have drawn a lot of attention recently for potential use in telecommunication devices, sensors, and lasers due to their high quality $(Q)$ factors. The high $Q$-factor resonances can be utilized in applications of channel add/drop filters, ${ }^{1,2}$ wavelength division multiplexing and demultiplexing, biosensors, ${ }^{3}$ and single-wavelength lasers. ${ }^{4}$ The $Q$ factor of a microring resonator is limited by optical losses, including surface roughness scattering, bending loss, coupling loss, and leakage loss. ${ }^{5}$ Previous research showed that the attainable $Q$ factor was primarily limited by surface roughness or imperfection, which scatters the guided wave into radiated modes. ${ }^{6}$ This loss mechanism greatly affects the performance of microring resonators fabricated in III-V semiconductors, dielectrics, silicon oxynitrides, and polymers. To solve this problem, Armani et al. have developed a laser annealing method to greatly reduce the surface roughness of a silica microdisk and demonstrated unprecedented $Q$ factors achieved in the silica microdisks. ${ }^{7}$

Recently, microring resonators have also been demonstrated in polymers. ${ }^{8,9}$ The polymer microring resonators possess such advantages as low cost, easy fabrication, and exploitation of high optical nonlinearities, where nonlinearities can be achieved in nonlinear optical polymers. ${ }^{9}$ Furthermore, polymers can have rich surface functionalities that are very attractive for potential biosensor applications. ${ }^{10}$ Like devices made in other materials, the surface roughness scattering is also the main loss factor for polymer microrings. In this letter, we propose a simple thermal-flow technique to reduce the surface roughness, and demonstrate its effectiveness in reducing the waveguide loss. In addition to smoothing surfaces, this technique simultaneously reduces the submicron gap separation between the waveguides, which improves the optical coupling and leads to more compact devices.

We have previously demonstrated polymer microring resonators fabricated by a direct imprinting technique. ${ }^{8}$ This technique can be applied to polymer waveguide devices from

${ }^{a)}$ Electronic mail: guo@eecs.umich.edu a wide range of thermal plastic materials such as polymethylmethacrylate, polystyrene (PS), and polycarbonate, to name only a few. Using this nanoimprint technique, polymer microring resonators are created by pressing a Si mold with inverse microring waveguide patterns [shown in Figs. 1(a) and 1(b)] under a high pressure and temperature into a PS thin film coated on an oxidized silicon wafer. During this process, the polymer layer is displaced and squeezed into the waveguide template on the mold. Figure 1(c) is a scanning electron microscopy (SEM) micrograph of an imprinted polymer device having a microring resonator coupled with two bus waveguides that act as the input, output, and drop ports. The waveguide has a rectangular cross section and sits on top of a pedestal structure that is etched into the underlying $\mathrm{SiO}_{2}$ layer to provide better light confinement. The oxide pedestals are formed by isotropic buffered HF etch. With respect to the optical characteristics, a microring resonator has a periodic resonance spectrum. A typical spectrum measured at the dropped port is shown in Fig. 1(d). Currently, the polymer microring resonators that we have fabricated can have $Q=\lambda_{c} / \delta \lambda \sim 10^{4}$, where $\lambda_{c}$ is the resonance wave-
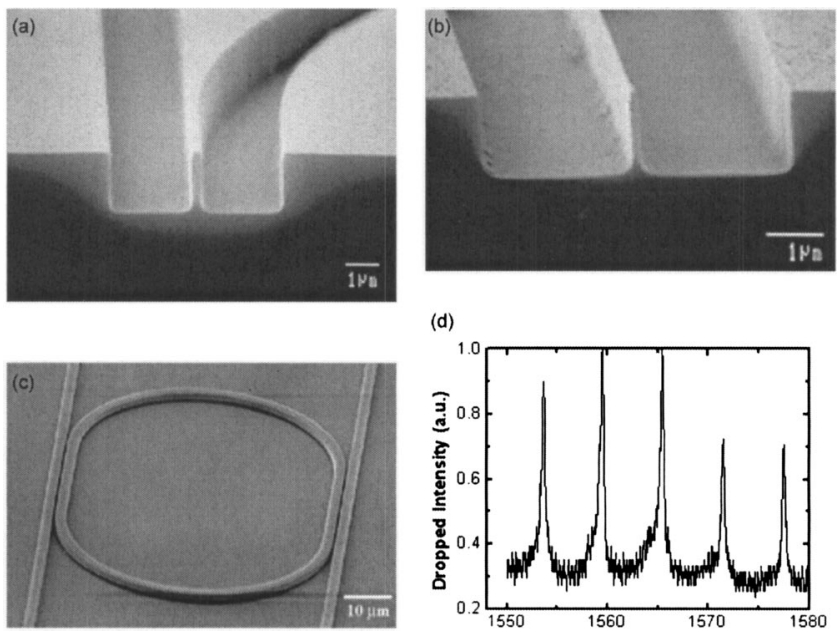

FIG. 1. SEM pictures of (a) $\mathrm{SiO}_{2}$ mold near the junction of the straight and the microring waveguides and (b) $\mathrm{SiO}_{2}$ mold in the coupling region. (c) An imprinted micro-racetrack resonator. (d) Typical spectrum of a PS microring resonator at the dropped ports. 


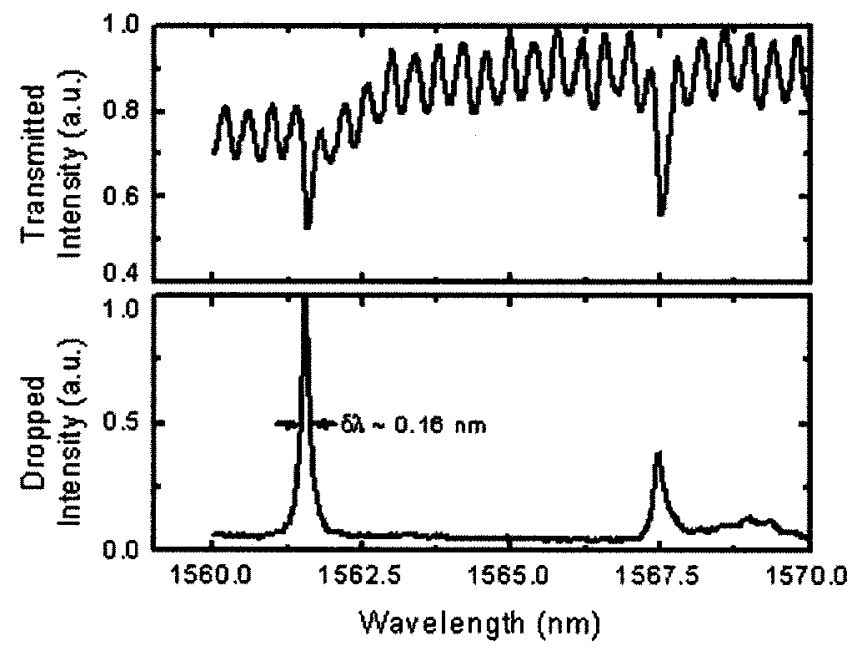

FIG. 2. The spectra of a PS microring resonator measured at the transmitted and drop ports with $Q$ factor around $10^{4}$ ( $Q$ defined as resonance wavelength divided by resonance linewidth).

length and $\delta \lambda$ the full width at half-maximum of the resonance peak. The optical spectra of such a device are shown in Fig. 2.

The thermal-flow processes are performed before the HF etching to form the pedestal structures. In this process, samples are heated up to various temperatures for different durations. This heat treatment reduces the viscosity of the polymer and enhances its fluidity, and the surface tension of the viscous polymer smoothes the surface corrugation of the waveguide. In order not to change the shape of the rectangular waveguide significantly, temperatures were chosen to be $10-\sim 20^{\circ} \mathrm{C}$ below the glass transition temperature of PS, $105^{\circ} \mathrm{C}$. For better comparison, we used the same mold to imprint several duplicated samples. The replicated polymer waveguides are conformal to the template on the mold, and thus preserve the same surface roughness as that on the mold. These samples are then annealed using different heating temperatures and times. SEM characterization [Figs. 3(a), 3(c), and 3(e)] shows the significantly reduced surface roughness after the thermal-flow process. Figure 3(a) shows the initial microring waveguide, Fig. 3(c) after $85^{\circ} \mathrm{C}$ annealing for $120 \mathrm{~s}$, and Fig. 3(e) after $95^{\circ} \mathrm{C}$ annealing for $60 \mathrm{~s}$. Within the temperature range that we have examined, higher temperature and longer heating time lead to smoother surfaces.

The thermal-flow process also deforms the waveguide profile. The waveguide shape obtained after annealing at different temperatures and times are illustrated in Figs. 3(b), $3(d)$, and 3(f), correspondingly. The original shape of the waveguide is a rectangle $2.3 \mu \mathrm{m}$ in width and $1.6 \mu \mathrm{m}$ in height. Annealing the samples rounds off the waveguide corners and increases its base width. This deformation changes slightly the effective refractive index of the guided mode that determines the resonant wavelengths, free spectra range, and bandwidth. However, as shown by these SEM pictures, the slight deformation is not expected to significantly influence the optical characteristics of microring resonators. On the other hand, if the heating temperature is too high and the heating time is too long, the waveguide shape can change dramatically from a rectangle to a mushroom shape. Hence,
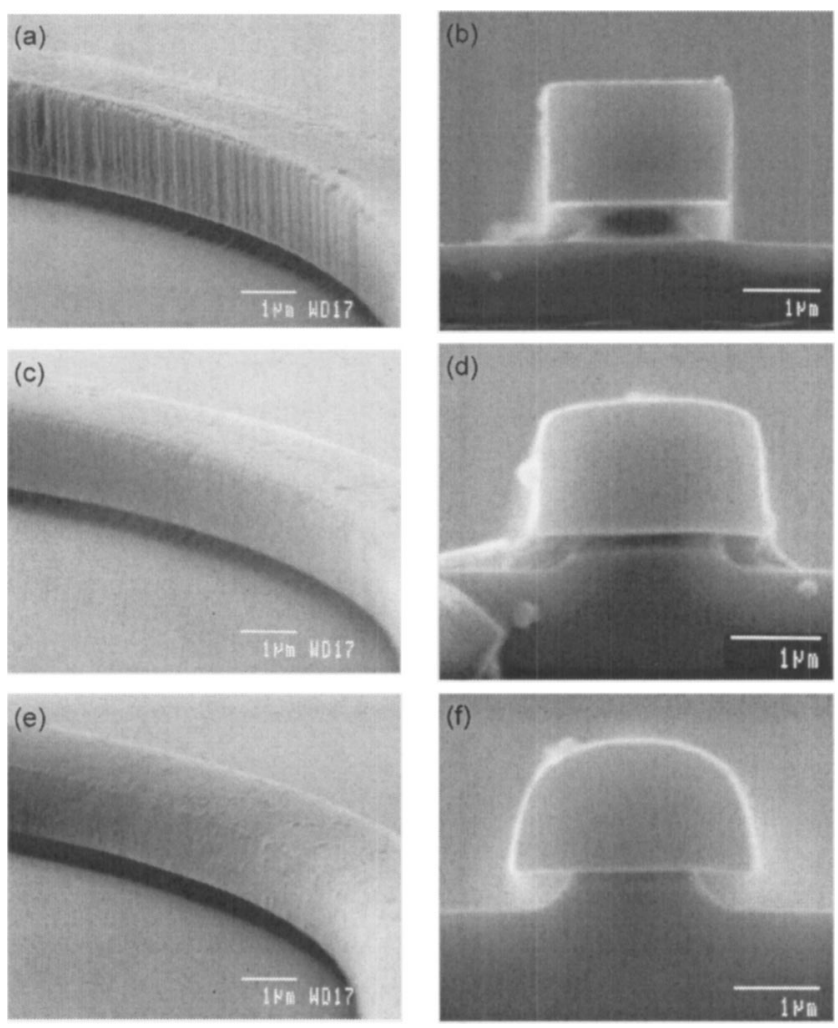

FIG. 3. SEM pictures showing the sidewall of microring waveguides before

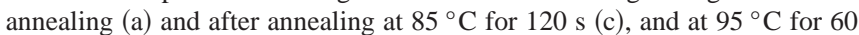
$\mathrm{s}(\mathrm{e})$. The corresponding cross-sectional SEM pictures are shown in (b), (d), and (f), respectively.

the heating temperature and time should be chosen carefully to avoid severe waveguide deformation.

To verify that the smoothed surface by the thermal-flow technique can reduce the propagation loss, we performed optical characterization on fabricated straight waveguides of various lengths. The waveguide loss can be extracted by using the Fabry-Perot contrast method. ${ }^{11}$ This method is based on the measured spectra of the Fabry-Perot waveguide resonator formed by the two cleaved end facets, and the propagation loss can be obtained from the contrast of the spectrum. Figure 4(a) shows an example of a measured interference spectrum for a 2.37-mm-long PS waveguide after $95^{\circ} \mathrm{C}$ annealing for $60 \mathrm{~s}$. A tunable laser is used as a light source and the light is coupled into the waveguide facet by using an objective lens. The waveguide loss is determined for different annealing parameters and they are shown in Fig. 4(b) as a function of the heating temperature. We observed a strong correlation between the reduction in the waveguide loss and the annealing temperatures. By annealing at $95^{\circ} \mathrm{C}$ for $60 \mathrm{~s}$, the propagation loss can be reduced by $9 \mathrm{~dB} / \mathrm{cm}$. On the other hand, annealing at $70^{\circ} \mathrm{C}$ for 3 min results in no morphology change verified by SEM, and thus no change in propagation loss is inferred. Clearly, the waveguide loss starts to decrease above a threshold temperature and is reduced further as higher temperatures are used. These results demonstrate that the thermal-flow technique can reduce the light scattering loss in polymer waveguide devices very effectively.

Another benefit of the thermal-flow technique is that it can simultaneously modify the gap distance between waveguides in the coupling region, which plays an important 


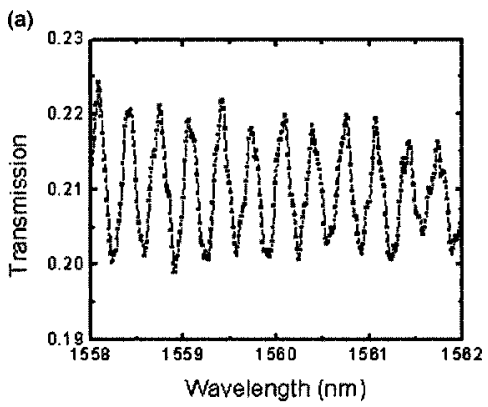

(b)

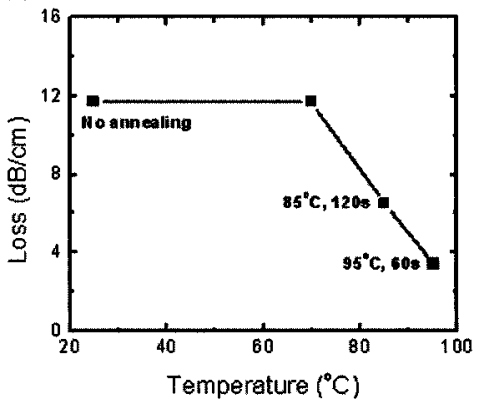

FIG. 4. (a) Fabry-Perot spectrum of a 2.37-mm-long PS waveguide after $95^{\circ} \mathrm{C}$ annealing for $60 \mathrm{~s}$. (b) Propagation losses versus the heating temperature for different annealing processes, indicating the effectiveness of thermal-flow technique in reducing the waveguide loss.

in microring resonators. The gap size determines optical properties of a microring, such as bandwidth of resonance, $Q$ factor, and contrast between high and low transmission. Depending on the coupling strength, the microring resonator device can operate in overcoupled, critically coupled, or undercoupled regimes. To obtain high contrast resonance, a microring resonator is preferred to operate at the critically coupled (propagation loss in the ring waveguide is equal to the amplitude transmission coefficient) or overcoupled condition in which sufficient coupling is needed in both cases. In order to obtain strong coupling, either a long coupling region or a narrow gap is required. However, long coupling length increases the device size, while a narrow gap in the submicron range increases the difficulty in fabrication. The thermal-flow technique can be used to effectively reduce the gap between two waveguides. Figure 5(a) illustrates the dependence of the change in gap width versus the annealing time at $90^{\circ} \mathrm{C}$. This figure shows that the reduction in gap width has a linear relationship to the heating time, indicating excellent control of the process. The inset of Fig. 5(a) shows the coupling region of a PS microring device after the annealing, and the coupling gap distance is reduced to $\sim 200 \mathrm{~nm}$ from the initial width of $\sim 450 \mathrm{~nm}$. Based on the coupled-mode theory, the amplitude cross-coupling coefficient $\kappa$ can be calculated, and is shown in Fig. 5(b) as a function of the gap separation. The coefficient can be enhanced by at least a factor of 3 . Such gap reduction by the simple thermal-flow technique can allow one to design a microring resonator with a wider initial gap than the optimal value and then gap is shrunk to the optimal size while surface roughness is reduced by the thermal-flow technique. This can be used to leverage the difficulty in producing the very narrow and high-aspect-ratio gaps in the device fabrication. Hence, combined with the reduction in surface roughness, the thermal-flow technique has the potential to ease the (a)

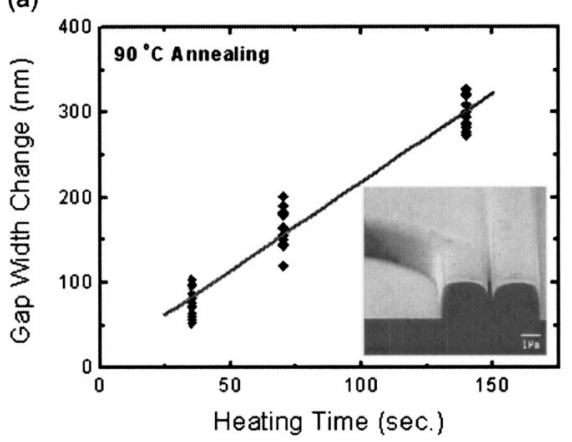

(b)

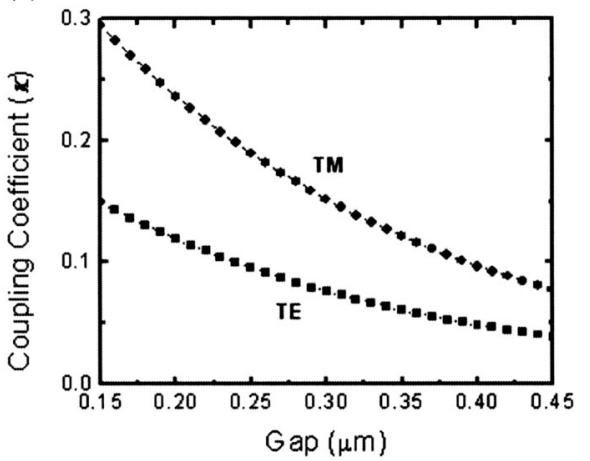

FIG. 5. (a) The reduction in gap width shows a linear relationship with the heating time at $90{ }^{\circ} \mathrm{C}$. The inset shows a PS microring resonator with the gap width of $\sim 200 \mathrm{~nm}$, reduced from the initial width of $450 \mathrm{~nm}$ by the thermal-flow technique. (b) The amplitude cross-coupling coefficient $\kappa$ for TE and TM polarizations increases with the reduction of the gap separation. The coupling length is fixed at $30 \mu \mathrm{m}$.

fabrication and greatly enhance the $Q$ factor of polymer microring resonators.

In conclusion, the thermal-flow technique can greatly smooth the surfaces and sidewalls of polymer waveguides, which reduces the light scattering and can be used to enhance the $Q$ factor of microring resonators. This concept is supported by SEM characterization and waveguide loss measurement. Furthermore, this technique is also able to shrink the gap separation in the coupling region of a microring resonator, which can lead to more compact devices and ease the fabrication difficulty of submicron gaps. In principle, the thermal-flow technique can be applied to many other polymer waveguide devices to achieve smoother surface and less scattering loss.

The authors thank NSF and DARPA for partial support of this work.

${ }^{1}$ B. E. Little, S. T. Chu, H. A. Haus, J. Foresi, and J.-P. Laine, J. Lightwave Technol. 15, 998 (1997).

${ }^{2}$ K. Djordjev, S. J. Choi, S. J. Choi, and P. D. Dapkus, IEEE Photonics Technol. Lett. 14, 828 (2002).

${ }^{3}$ E. Krioukov, D. J. W. Klunder, A. Driessen, J. Greve, and C. Otto, Opt. Lett. 27, 512 (2002).

${ }^{4}$ B. Liu, A. Shakouri, and J. E. Bowers, IEEE Photonics Technol. Lett. 14, 600 (2002).

${ }^{5}$ V. Van, P. P. Absil, J. V. Hryniewicz, and P.-T. Ho, J. Lightwave Technol. 19, 1734 (2001).

${ }^{6}$ V. Van, P. P. Absil, J. V. Hryniewicz, and P.-T. Ho, Technical Digest of CLEO, 2001, p. 263.

${ }^{7}$ D. K. Armani, T. J. Kippenberg, S. M. Spillane, and K. J. Vahala, Nature (London) 421, 925 (2003).

${ }^{8}$ C. Y. Chao and L. J. Guo, J. Vac. Sci. Technol. B 20, 2862 (2002).

${ }^{9}$ P. Rabiei, W. H. Steier, C. Zhang, and L. R. Dalton, J. Lightwave Technol. 20, 1968 (2002).

${ }^{10}$ C. Y. Chao and L. J. Guo, Appl. Phys. Lett. 83, 1527 (2003).

${ }^{11}$ R. J. Deri and E. Kapon, IEEE J. Quantum Electron. 27, 626 (1991). 